\title{
Directed evolution and targeted mutagenesis to murinize listeria monocytogenes internalin A for enhanced infectivity in the murine oral infection model
}

\author{
Ian R Monk ${ }^{1,3}$, Pat G Casey ${ }^{1}$, Colin Hill ${ }^{1 *}$, Cormac GM Gahan²
}

\begin{abstract}
Background: Internalin A ( $|\mathrm{n}| \mathrm{A})$ is a critical virulence factor which mediates the initiation of Listeria monocytogenes infection by the oral route in permissive hosts. The interaction of InIA with the host cell ligand E-cadherin efficiently stimulates L. monocytogenes entry into human enterocytes, but has only a limited interaction with murine cells.

Results: We have created a surface display library of randomly mutated InIA in a non-invasive heterologous host Lactococcus lactis in order to create and screen novel variants of this invasion factor. After sequential passage through a murine cell line (CT-26), multiple clones with enhanced invasion characteristics were identified. Competitive index experiments were conducted in mice using selected mutations introduced into $L$. monocytogenes EGD-e background. A novel single amino acid change was identified which enhanced virulence by the oral route in the murine model and will form the basis of further engineering approaches. As a control a previously described EGD-InIA ${ }^{m}$ murinized strain was also re-created as part of this study with minor modifications and designated EGD-e InIA ${ }^{m *}$. The strain was created using a procedure that minimizes the likelihood of secondary mutations and incorporates Listeria-optimized codons encoding the altered amino acids. L. monocytogenes EGD-e InIA ${ }^{m *}$ yielded consistently higher level murine infections by the oral route when compared to EGD-e, but did not display the two-fold increased invasion into a human cell line that was previously described for the EGD-InIA ${ }^{\mathrm{m}}$ strain.

Conclusions: We have used both site-directed mutagenesis and directed evolution to create variants of InIA which may inform future structure-function analyses of this protein. During the course of the study we engineered a murinized strain of L. monocytogenes EGD-e which shows reproducibly higher infectivity in the intragastric murine infection model than the wild type, but does not display enhanced entry into human cells as previously observed. This murinized L. monocytogenes strain will provide a useful tool for the analysis of the gastrointestinal phase of listeriosis.
\end{abstract}

\section{Background}

Internalin A (InlA) is a sortase achored, cell wall protein and a critical factor in the pathogenesis of the foodborne Gram-positive pathogen Listeria monocytogenes. InlA stimulates L. monocytogenes entry into normally non-phagocytic intestinal enterocytes [1]. The protein is

\footnotetext{
* Correspondence: c.hill@ucc.ie

'Alimentary Pharmabiotic Centre \& Department of Microbiology, University College Cork, Western Road, Cork, Ireland

Full list of author information is available at the end of the article
}

800 amino acids (aa) in length and composed of seven distinct domains (Figure 1a); (i), 1-35 aa: a consensus Nterminal signal sequence (SS); (ii), 35-78 aa: forms a cap at the N-terminus tip of mature protein (C); (iii), 79-407 aa: 15 Leucine rich repeats (LRR) with 14 containing 22 aa (repeat 6 contains 21 aa) (shaded grey); (iv), 415-495 aa: an inter-repeat domain (IR); (v), 518-706 aa: three $\beta$ repeat domains, which may serve as a stalk to project the sickle shaped LRR out from the cell surface $(\beta 1, \beta 2$ and $\beta 3$ ); and (vi), 767-771 aa: a sortase cleavage site 


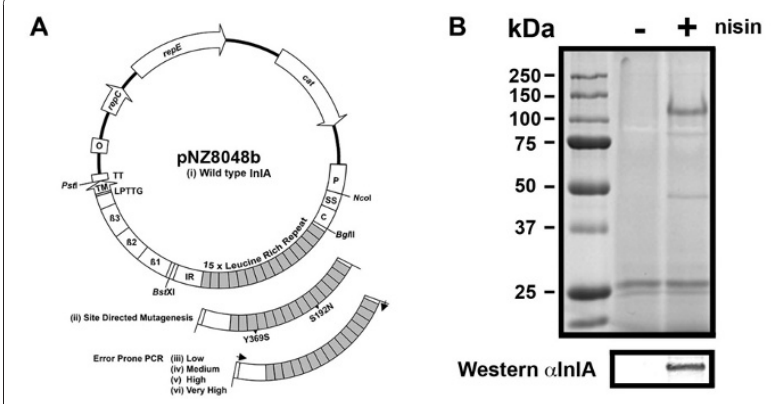

Figure 1 Nisin inducibe InlA plasmid constructs and the expression of InIA ${ }^{W T}$ on the surface of $L$. lactis. A. Lactococcal nisin inducible plasmid pNZB with the entire (i) in/ $A^{\mathrm{WT}}$ gene from EGD-e cloned upstream of the nisin inducible nisA promoter (P). The labels for the InIA domains are described in the introduction text. The naturally occuring $B g / l / B s t X \mid$ restriction sites within the inlA gene encompass the entire $L R R$ required for the interaction with $E$ cadherin. These two sites were used for the cloning of the (ii) the murinized version of in $/ A\left(\right.$ in $\left./ A^{m_{*}}\right)$ with the amino acid changes as described by Wollert et al. [17] (created by site directed mutagenesis) and (iii) four randomly mutated banks of in/A generated by error prone PCR. B. A lysozyme cell wall extract was isolated from L. lactis $\operatorname{InI} \mathrm{A}^{\mathrm{WT}}$ grown under the conditions used for invasion assay. Exponential phase cells $(O D=0.6)$ were cultured for $1 \mathrm{~h}$ in the presence (+) or absence (-) of nisin (approx $10 \mathrm{ng} / \mathrm{ul}$ ). Proteins were run on 10\% SDS-PAGE and either stained with coomassie blue or blotted and detected with a InIA monoclonal antibody [23].

(LPPTG) for peptidoglycan cross linking and (vii) 771-800 aa: a membrane targeting sequence (TM)[2]. Domain (iii) containing the LRR's of InlA is sufficient to stimulate enterocyte uptake $[3,4]$. The enterocyte ligand for InlA was identified as E-cadherin (CDH1) [5], which is required by host cells for the formation of tight junctions and to promote cellular polarization, communication and differentiation [6]. The localization of CDH1 on the basolateral face of differentiated cells suggested that invasion was a secondary event, occurring after non-specific uptake by $\mathrm{M}$ cells [5]. Oral infection studies using rats [7] and mice [8] provided support for this hypothesis. However, oral infections resulted in the invasion of enterocytes in a guinea pig model [9]. Human colonic Caco-2 enterocyte cells are also directly permissive to infection in vitro $[9,10]$. These seemingly anomalous results are due to the reduced affinity of murine CDH1 (mCDH1) for InlA. The reduced affinity was localized to amino acid 16 which is a proline in guinea pig and human CDH1 (hCDH1) but in rats and mice a glutamic acid is present [11]. This discovery led to the development and application of a transgenic mouse model expressing both human and murine CDH1 within intestinal enterocytes, which conclusively demonstrated the role of InlA in the pathogenesis of orally acquired L. monocytogenes [12]. In an elegant study, the site of enterocyte cell extrusion at the tips of intestinal villi was identified as a mechanism for exposing $\mathrm{CDH} 1$ on the apical surface at multicellular junctions [13]. More recently, a transgenic mouse strain that ubiquitously expresses human E-cadherin has been developed to demonstrate a role for InlA (and InlB) in fetoplacental listeriosis [14].

The crystal structure of InlA in complex with hCDH1 demonstrated the structural importance of proline 16 for the interaction [15]. In silico analysis confirmed that the reduced affinity of InlA for mCDH1was essentially due to the steric hindrance imposed by the bulky glutamic acid at aa 16, which therefore could not interact with the hydrophobic pocket (between LRR's 5, 6 and 7 of InlA) created by the removal of one amino acid from LRR 6 [15]. Overall the crystal structure identified 28 residues of hCDH1 that interact with the residues across the LRR region. Structural data and the invasion results from previous research $[3,4]$ have confirmed the essential nature of the LRR's in the InlA:: CDH1 interaction.

Small animal model of listeriosis have a number of significant limitations. Even though rabbits and guinea pigs possess a permissive CDH1, they have recently been shown to be resistant to systemic infection due to a species specificity observed in the InlB/host interaction [16]. InlB is required for efficient hepatocyte/endothelial cell invasion in the mouse model and in certain human cell lines. A novel approach to address the lack of appropriate animal models focused on the 'murinization' of L. monocytogenes rather than the 'humanization' of mice [17]. Rational protein design based on the structural data of the InlA/hCDH1 complex, identified two mutations in InlA (Ser192Asn and Tyr369Ser) that dramatically increased the affinity for both hCDH1 and $\mathrm{mCDH} 1$. This allowed the development of a variant of L. monocytogenes EGD-e (EGD-InlA ${ }^{\mathrm{m}}$ ) capable of establishing systemic infections in C57BL/6J mice after oral inoculation [17]. However, the strain also exhibited a 2fold increase in adhesion and consequently invasion into human cells, suggesting that the alteration in tropism towards mice also could enhance the virulence towards humans.

To address any remaining concerns regarding human virulence of murinized $L$. monocytogenes, we conducted random mutagenesis of InlA combined with surface display on a non-invasive, Gram-positive, Lactococcus lactis to identify mutations that improve the entry into a colonic murine cell line. Using the CT-26 cells as a selection tool, multiple positive mutations in independent clones were identified with an enrichment in the InlA/hCDH1 interacting residues. The inlA genes from $4 \mathrm{~L}$. lactis clones were separately recombined into the inlA chromosomal locus in EGD-e $\Delta i n l A$ generating EGD-e A to D. Also, a version of EGD-InlA ${ }^{\mathrm{m}}$ [17] was created in order to permit comparison with our newly generated 
InlA mutant strains. In contrast to the strategy employed by Wollert et al. [17] we utilised preferred Listeria codons for the mutated 192Asn and 369Ser and designated the strain; EGD-e InlA ${ }^{m *}$. Strains were competed against EGD-e InlA ${ }^{m *}$ in oral murine competitive index assays [18]. A novel aa mutation was identified which enhanced InlA/mCHD1 interaction compared to EGD-e. In agreement with earlier studies [17] the adherence/invasion into Caco-2 cells and virulence by murine intravenous infection of the codon-optimized EGD-e InlA $^{m *}$ strain was indistinguishable from EGD-e, while EGD-e InlA $^{m *}$ alone exhibited highly reproducible murine oral infections.

\section{Methods}

\section{Bacterial and Cell Culture}

Bacterial strains, plasmids and oligonucleotides are described in Table 1. For the routine propagation of L. lactis MG1363 derivative NZ9000, cells were grown statically at $30^{\circ} \mathrm{C}$ in M17 (Oxiod) broth containing $0.5 \%$ $\mathrm{w} / \mathrm{v}$ filter sterilized glucose (GM17). L. monocytogenes were cultivated in BHI (Oxiod) and Escherichia coli grown in $\mathrm{LB}$ at $37^{\circ} \mathrm{C}$ with shaking at $200 \mathrm{rpm}$. For growth on agar, respective broths were solidified with $1.5 \%(\mathrm{w} / \mathrm{v})$ agar (Merck). For blue/white screening in $L$. monocytogenes, X-gal (Merck) was incorporated into $\mathrm{BHI}$ agar at $100 \mu \mathrm{g} / \mathrm{ml}$. Antibiotics were added when required: erythromycin E. coli - $250 \mu \mathrm{g} / \mathrm{ml}$, L. monocytogenes - $5 \mu \mathrm{g} / \mathrm{ml}$ and chloramphenicol L. lactis - $5 \mu \mathrm{g} / \mathrm{ml}$. Plasmids were isolated from NZ9000 after overnight growth in $10 \mathrm{ml}$ of GM17. To lyse, the pellet was resuspended in $500 \mu \mathrm{l}$ of P1 buffer (see Qiagen manual) containing $30 \mu \mathrm{g}$ of lysozyme and incubated for $30 \mathrm{~min}$ at $37^{\circ} \mathrm{C}$. The lysate was processed as described in the Qiaprep spin miniprep kit (Qiagen). A nisin filtrate for PnisA induction was isolated from the supernatant of an overnight L. lactis culture of NZ9700 (filter sterilized through $0.22 \mu \mathrm{M}$ low protein binding filters - Millipore), aliquots frozen at $-20^{\circ} \mathrm{C}$. For all InlA induction experiments, overnight L. lactis NZ9000 cultures (containing pNZ8048 plasmids) were diluted 1:20 in $10 \mathrm{ml}$ of fresh GM17 and grown to an $\mathrm{OD}_{600 \mathrm{~nm}}$ of 0.5 (approximately $2 \mathrm{~h}$ ). The expression of $i n l A$ was induced with $10 \mu \mathrm{l}$ of nisin and grown for a further hour to an OD $=1.0\left(5 \times 10^{8} \mathrm{cfu} / \mathrm{ml}\right)$. The murine (CT-26) and human (Caco-2) colonic epithelial cell lines were routinely cultured at $37^{\circ} \mathrm{C}$ in $5 \% \mathrm{CO}_{2}$. Media was composed of DMEM glutamax, 10\% FBS, Pen/ Strep and 1\% non essential amino acids with all cell culture media purchased from Gibco. Oligonucelotides were purchased from Eurofins MWG Operon.

\section{Production of electrocompetent Lactococcus lactis}

The protocol of Holo and Nes [19] was adapted for the transformation of L. lactis MG1363 derivative NZ9000.
A GM17 overnight culture of NZ9000 was diluted 1:100 into $5 \mathrm{ml}$ of GM17 containing $500 \mathrm{mM}$ sucrose and $2.5 \%$ glycine (GS-GM17). This culture was inoculated into $50 \mathrm{ml}$ of fresh GS-GM17 and grown overnight. The $50 \mathrm{ml}$ culture was inoculated into $400 \mathrm{ml}$ of fresh GS-GM17, grown to OD600 of 0.3 and cells were subsequently harvested by centrifugation at $4,000 \times \mathrm{g}$ for $20 \mathrm{~min}$ at $4^{\circ} \mathrm{C}$. The pellet was resuspended in 200 $\mathrm{ml}$ of ice cold SGB (500 mM sucrose and 10\% (w/v) glucose - filter sterilized), centrifuged, resuspended in $100 \mathrm{ml} \mathrm{SGB}$ and left on ice for $15 \mathrm{~min}$. The cells were centrifuged, resuspended in $50 \mathrm{ml} \mathrm{SGB}$ and left on ice for 15 min before a final centrifugation and re-suspension with $2 \mathrm{ml} \mathrm{SGB}$. Cells were frozen at $-80^{\circ} \mathrm{C}$ in 40 $\mu \mathrm{l}$ aliquots. To electroporate, cells were thawed on ice, mixed with $4 \mathrm{ul}$ of pellet paint (Novagen) precipitated DNA and transferred to a $1 \mathrm{~mm}$ electroporation cuvette (Biorad). Cells were pulsed at $20 \mathrm{kV} / \mathrm{cm}, 200 \Omega$ and $25 \mu \mathrm{F}$, regenerated in $1 \mathrm{ml} \mathrm{GM} 17$ containing 2 $\mathrm{mM} \mathrm{CaCl} 2 / 20 \mathrm{mM} \mathrm{MgCl} 2$ for $1.5 \mathrm{~h}$ and then plated onto GM17 agar containing $5 \mu \mathrm{g} / \mathrm{ml}$ chloramphenicol. An efficiency of $1 \times 10^{7} \mathrm{cfu} / \mu \mathrm{g}$ was routinely obtained with pNZ8048.

\section{Cloning of InIA into pNZB}

The unique $B g l$ II site up stream of the nis $A$ promoter in pNZ8048 was removed by linearization of the vector with BglII and ends blunted with T4 DNA polymerase. The vector was religated to generate $\mathrm{pNZB}$. The inlA gene was PCR amplified (primers IM194 and IM188) as described previously [20], digested with NcoI/PstI and ligated into the complementary digested pNZB. Ligations were directly electroporated into NZ9000 as described above and the sequence of the inlA gene was verified by DNA sequencing.

\section{QuikChange mutagenesis in L. lactis}

Primers for site directed mutagenesis (SDM) (Table 1) were designed according to the Quikchange SDM manual (Stratagene). All plasmid template isolated from NZ9000 strains was methylated with Dam methylase following manufacturer recommendations (New England Biolabs). The PCR thermocycling conditions were conducted as described previously [21]. Separate $50 \mu \mathrm{l} \mathrm{KOD}$ hotstart high fidelity polymerase PCR reactions were preformed with each primer for 10 cycles and an extension time of $5 \mathrm{~min} 30 \mathrm{sec}$. After 10 cycles the reactions were combined and continued for an additional 18 cycles. Amplimers were column purified (Qiaquick PCR purification kit, Qiagen) and digested overnight with $D p n I$ (Roche). Digests were pellet paint precipitated and the half of the digest directly electroporated into NZ9000. Between 200 and 1000 colonies were obtained per transformation. The protocol was repeated to 
Table 1 Bacterial strains, plasmids and oligonucleotides

\begin{tabular}{|c|c|c|}
\hline Name & Description & Source \\
\hline \multicolumn{3}{|l|}{ Bacterial strains } \\
\hline EC10B & E. coli $\mathrm{DH} 10 \mathrm{~B}$ derivative, with repA integrated into the glgB gene. Kan'. & [20] \\
\hline NZ9000 & Nisin responsive L. lactis MG1363 derivative, with nisRK integrated into the pepN gene. & [26] \\
\hline EGD-e & L. monocytogenes 1/2a strain. Genome sequenced. Obtained from Werner Goebel. & [39] \\
\hline EGD-e $\triangle \operatorname{in} / A$ & EGD-e with the E-cadherin interacting region of InIA deleted (amino acids 80 to 506) & [20] \\
\hline $\begin{array}{l}\text { EGD-e\in/A:: } \\
\text { pIMK2in/A }\end{array}$ & EGD-e $\triangle$ inlA with InlA over expressed from the Phelp promoter integrated at tRNA ${ }^{A r g}$ locus, Kan ${ }^{r}$ & [20] \\
\hline EGD-e $\ln \mid A^{m *}$ & EGD-e with in/A residues S192N and Y369 S modified in the chromosome. & This study \\
\hline EGD-e A & EGD-e $\triangle$ in/A with in/A locus recreated containing SDM change N259Y in the chromosome. & This study \\
\hline EGD-e B & EGD-e $\Delta$ in/A with in/A locus recreated containing SDM change Q190L in the chromosome. & This study \\
\hline EGD-e C & EGD-e $\Delta$ in/A with in/A the locus recreated containing SDM changes S173I, L185F and L188F in the chromosome. & This study \\
\hline EGD-e D & EGD-e $\triangle$ in/A with in/A locus recreated containing SDM changes T164A, K301I and G303E in the chromosome. & This study \\
\hline $\begin{array}{l}\text { EGD-e InlA } \text { m }_{*}:: \\
\text { plMC3ery }\end{array}$ & EGD-e $\operatorname{In|} A^{m_{*}^{*}}$ with the IPTG inducible expression of erythromycin integrated in the tRNA ${ }^{A R G}$ locus, $\mathrm{Cm}^{r}$. & This study \\
\hline EGD-e::pIMC3kan & EGD-e with the IPTG inducible expression of kanamycin integrated in the tRNA ${ }^{A R G}$ locus, $\mathrm{Cm}^{r}$. & [18] \\
\hline $\begin{array}{l}\text { EGD-e A:: } \\
\text { pIMC3kan }\end{array}$ & EGD-e A with the IPTG inducible expression of kanamycin integrated in the tRNA ${ }^{A R G}$ locus, $\mathrm{Cm}^{r}$ & This study \\
\hline $\begin{array}{l}\text { EGD-e B:: } \\
\text { pIMC3kan }\end{array}$ & EGD-e B with the IPTG inducible expression of kanamycin integrated in the tRNA ${ }^{A R G}$ locus, $\mathrm{Cm}^{r}$ & This study \\
\hline $\begin{array}{l}\text { EGD-e C:: } \\
\text { pIMC3kan }\end{array}$ & EGD-e $C$ with the IPTG inducible expression of kanamycin integrated in the tRNA ${ }^{A R G}$ locus, $\mathrm{Cm}^{r}$ & This study \\
\hline $\begin{array}{l}\text { EGD-e D:: } \\
\text { pIMC3kan }\end{array}$ & EGD-e D with the IPTG inducible expression of kanamycin integrated in the tRNA ${ }^{A R G}$ locus, $\mathrm{Cm}^{r}$ & This study \\
\hline NZ9700 & Nisin producer, progeny of NIZO B8 and MG1363 (Rif' and Strp') conjugation. & [26] \\
\hline
\end{tabular}

Plasmids

pNZB

pNZBinIA ${ }^{W T}$

Nisin inducible plasmid with heterologous gene expressed from the nis $A$ promoter. $B g$ /ll site upstream of nis $A$ removed.

pNZBinlA ${ }^{m *}$

Internalin A from EGD-e containing the entire gene including signal sequence. Cloned into Ncol/Pstl of pNZB.

This study

pNZBin/A Bank-iii Internalin A containing S192N and Y369 S in pNZB.

pNZBin/A Bank-iv

Error Prone PCR with low level of mutation 0-4.5 nt per kb.

Error Prone PCR with medium level of mutation 4.5-9 nt per kb.

pNZBinlA Bank-v Error Prone PCR with high level of mutation 9-16 nt per kb.

pNZBin/A Bank-vi Error Prone PCR with very high level of mutation 9-16 nt per kb.

PORI280 RepA negative gene replacement vector, constitutive lacZ, $5.3 \mathrm{~kb}, \mathrm{Em}$ '.

This study

This study

This study

This study

This study

This study

[40]

pORI280inIA(SDM) PCR amplified mutated inlA ${ }^{m_{*}}$ into pORI280 as Ncol/Pstl fragment. Contains wild type inlA promoter. This study

pORI280in/A(A) PCR amplified mutated in/A (from bank $v$ clone 6 containing N259Y) into pORI280 as Ncol/Pstl fragment. Contains This study Wt inlA promoter.

pORI280in/A(B) PCR amplified mutated in/A (from bank iii clone 3 containing Q190L) into pORI280 as Ncol/Pstl fragment. Contains This study Wt inlA promoter.

pORI280in/A(C) PCR amplified mutated in/A (from bank $v$ clone 6 containing S173I, L185F, L188F) into pORI280 as Ncol/Pstl This study fragment. Contains Wt inlA promoter.

pORI280in/A(D) PCR amplified mutated in/A (from bank $v$ clone 8 containing T164A, K301l, G303E) into pORI280 as Ncol/Pstl This study fragment. Contains Wt inlA promoter.

pVE6007 Temperature-sensitive helper plasmid, supplies RepA in trans. $\mathrm{Cm}^{\mathrm{r}}$.

[41]

\begin{tabular}{llc}
\hline Name & Oligonucleotide sequence $\left(\mathbf{5}^{\prime}-\mathbf{3}^{\prime}\right)^{\boldsymbol{a}}$ & $\begin{array}{c}\text { Restriction } \\
\text { site }\end{array}$ \\
\hline IM194 (inIA-F) & ATATCCATGGAAAAAACGATATGTATGGTTG & Ncol \\
IM188 (inIA-R) & TITCTGCAGTTATTACTAGCACGTGCTTTITAG & PStI \\
IM345 (S192N & CAGGTTAACTAGTCTACAGCAATTAAATTTGGTAATCAAGTGACAGATTAAAACC &
\end{tabular}

SDM-F)

IM346 (S192N

SDM-R)

IM349 (Y369 S CAAAGCTTCAAAGATTATTITTCTCTAATAACAAGGTAAGTGACGTAAG

SDM-F) 
Table 1 Bacterial strains, plasmids and oligonucleotides (Continued)

\begin{tabular}{|c|c|c|}
\hline $\begin{array}{l}\text { IM350 (Y369 S } \\
\text { SDM-R) }\end{array}$ & CTTACGTCACTTACCTTGTTATTAGAGAAAAATAATCTTTGAAGCTTTG & \\
\hline $\begin{array}{l}\text { IM490 } \\
\text { (Chromosome-F) }\end{array}$ & ATATCCATGGAAAAGGAGTGTATATAGTGAGAAAAAAACGATATGTATGG & Ncol \\
\hline $\begin{array}{l}\text { IM4666 } \\
\text { (Chromosome-R) }\end{array}$ & ATATCTGCAGCAAACGTTGCTGTATAGCTATTGG & Pstl \\
\hline IM467 (inlA out-F) & TATATAGGAAAAATGTGCTGGAACG & \\
\hline IM468 (in|A out-R) & TCCTTGATAGTCTACTGCTTGAGTCG & \\
\hline IM317 (in|A muta-F) & AAACAGATCTAGACCAAGTTACAACG & Bg/ll \\
\hline IM318 (in|A muta-R) & AATTCCACTTCTTTGGTTGTTCTTTGC & BstXI \\
\hline
\end{tabular}

${ }^{a}$ Restriction sites are highlighted in bold. Mutated triplets are underlined. The start codon of inlA is in italics.

combine SDM changes. From the final mutagenized plasmids, BglII/BstXI fragments containing the LRR region of InlA were excised and ligated into complementary digested pNZBinlA ${ }^{\mathrm{WT}}$.

\section{Isolation of cell wall proteins}

Cell wall proteins were isolated from nisin induced 10 $\mathrm{ml}$ NZ9000+pNZBinlA ${ }^{\mathrm{WT}}$ culture as described by previously [22], except cells were rendered as protoplasts for $1 \mathrm{hr}$ at $30^{\circ} \mathrm{C}$ without mutanolysin. Blotted proteins were probed with the InlA specific monoclonal antibody described by Hearty et al [23].

\section{Random Bank of inlA mutants in NZ9000}

The generation of a randomly mutated inlA bank between amino acids 74 and 512 (containing the LRR) of InlA was accomplished by error prone PCR with Mutazyme II (Stratagene). Plasmid DNA (pNZBinla ${ }^{\mathrm{WT}}$ ) was used as template in the reaction (primers IM317 and IM318) and a $1.3 \mathrm{~kb}$ fragment amplified between two naturally occurring restriction sites (BglII and $B s t \mathrm{XI}$ ). From the mutagenesis reactions, four different mutation rates by varying the amount of template used ((iii) $1000 \mathrm{ng}$ (iv) $250 \mathrm{ng}$ (v) $10 \mathrm{ng}$ and (vi) $0.1 \mathrm{ng}$ ). This equates to a sliding scale of increasing mutation frequency. Each amplimer pool was digested with BglII and BstXI and ligated into complementary digested pNZ8048binlA. The ligations (100 ng of pNZB with 240 ng of $i n l A$ ) were pellet paint precipitated and electroporated into electrocompetent NZ9000 (repeated twice). For each pool a total of 40,000 colonies were obtained with plasmid religations accounting for $0.125 \%$ of the total (about 50 colonies per 40,000). The colonies from each mutation frequency were pooled and frozen at $-80^{\circ}$ C. From each mutation frequency, 10 individual colonies were subjected to plasmid isolation as described above and the mutated region sequenced to access the level of mutagenesis.

\section{CT-26 and Caco-2 invasion assays}

Overnight cultures of NZ9000 containing pNZB only or pNZBinlA derivatives (Figure 1a) were induced as described above. A one $\mathrm{ml}$ aliquot was then pelleted at $4,000 \times g$ for $5 \mathrm{~min}$ and resuspended in $1 \mathrm{ml}$ of DMEM. Cells were centrifuged, resuspended in fresh DMEM and then diluted to a multiplicity of infection of 25:1. L. monocytogenes cells were grown as described previously prior to invasion [20]. CT-26 [24] and Caco-2 cells were seeded at $2 \times 10^{4}$ and $1 \times 10^{5}$ cells, respectively and grown for 4 days until confluency in 24 well plates (Falcon). On the day prior to use, antibiotics were removed from the media. On the day of use, cells were washed twice with DMEM to remove FBS. Both cell types were invaded for $1 \mathrm{~h}$ at $37^{\circ} \mathrm{C}$ in $5 \% \mathrm{CO}_{2}$, washed once with Dulbecco's PBS (Sigma) and then overlayed with DMEM containing 10 (Caco-2) or $100 \mu \mathrm{g} / \mathrm{ml}$ (CT-26) gentamicin for $1 \mathrm{~h}$. Monolayers were washed a further three times with PBS to remove residual antibiotic and then lysed with $1 \mathrm{ml}$ of ice cold sterile water. Bacterial cells were enumerated by serial dilution in PBS and plated on GM17 agar containing $5 \mu \mathrm{g} / \mathrm{ml}$ chloramphenicol. The remaining lysate from error prone PCR pools were inoculated into GM17 containing $5 \mu \mathrm{g} / \mathrm{ml}$ chloramphenicol, grown overnight, stocked at $-80^{\circ} \mathrm{C}$ with the protocol repeated for seven passages through CT-26 cells. EGD-e derivatives were plated onto $\mathrm{BHI}$ agar.

\section{Internalin A chromosomal mutagenesis in $L$. monocytogenes}

A $2 \mathrm{~kb}$ fragment was PCR amplified (primers IM467 and IM490) from the appropriate mutated pNZ8048binlA plasmid, with primer design incorporating the first $16 \mathrm{nt}$ upstream of the inlA GTG start codon. The amplimers were digested with $\mathrm{NcoI} /$ Pst I, ligated into complementary digested pORI280 and transformed into E. coli strain EC10B (Table 1). The plasmids pORI280 and pVE6007 we co-transformed into EGD-e $\Delta i n l A$ and mutagenesis 
preformed as described by previously [20]. The reconstruction of the inlA locus was identified by colony PCR (primers IM317 and IM318) with the integrity of the gene confirmed by DNA sequencing.

Intragastric versus intravenous infections of Balb/c mice For all murine experiments, 6-8 week old female Balb/c mice (Harlan) were used. All experiments were approved by the institutional ethics committee. Tail vein intravenous infections were conducted as described previously [18] with an inoculum comprised of equal numbers of EGD-e::pIMC3kan and EGD-e InlA ${ }^{m *}::$ pIMC3ery $\left(2 \times 10^{4}\right.$ total in $\left.100 \mu \mathrm{l}\right)$. For oral inoculation, overnight cultures were centrifuged $(7,000 \times$ g for 5 min), washed twice with PBS and resuspended at $5 \times$ $10^{10} \mathrm{cfu} / \mathrm{ml}$ in PBS containing $100 \mathrm{mg} / \mathrm{ml}$ of $\mathrm{CaCO}_{3}$. A $200 \mu \mathrm{l}$ inoculum was comprised of either a single strain $\left(5 \times 10^{9} \mathrm{cfu}\right)$ or a two strain mixture $\left(5 \times 10^{9}\right.$ of each strain). Mice were intragrastrically gavaged and the progression of infection followed over a three day time course. For bioluminescent imaging, mice were anesthetized on day 1 through to day 3 with isoflurane gas and imaged in a Xenogen IVIS 100 (Xenogen) at a binning of 16 for $5 \mathrm{~min}$. Mice were euthanized with spleen and livers aseptically removed, imaged (binning of 8 for 5 min) and enumerated as previously described [18].

\section{Results}

\section{A $L$. monocytogenes gentamicin protection assay for murine cells}

Invasion into Caco-2 cells by $L$. monocytogenes is dependent on the expression of functional InlA [10]. We confirmed that a $L$. monocytogenes mutant producing InlA without the LRR and IR domain $(\triangle i n l A)$ is severely compromised in invasion, while an over expressing InlA strain exhibits dramatically enhanced invasion (Figure 2). To establish an equivalent murine assay for $L$. monocytogenes we used monolayers of CT-26 cells (murine colonic carcinoma cell line) originally isolated from Balb/c mice chemically treated to induce tumor formation [24]. While CT-26 cells are not enterocyte like (they exhibit an undifferentiated-fibroblast appearance [25]), the results from invasion assays showed that they provide characteristics suitable for use as an invasion model (Figure 2). The $\triangle i n l A$ strain displayed a slight reduction (not statistically significant) in invasion compared to EGD-e, while over expression of InlA resulted in a modest increase in invasion. We speculate that this is due to a reduced affinity of InlA for $\mathrm{mCDH} 1$, however we have not assayed for $\mathrm{mCDH} 1$ production by CT- 26 cells.

Heterologous expression was then employed to distinguish InlA from additional virulence determinants on the surface of the $L$. monocytogenes. We chose to use the well characterized nisin inducible expression system [26]

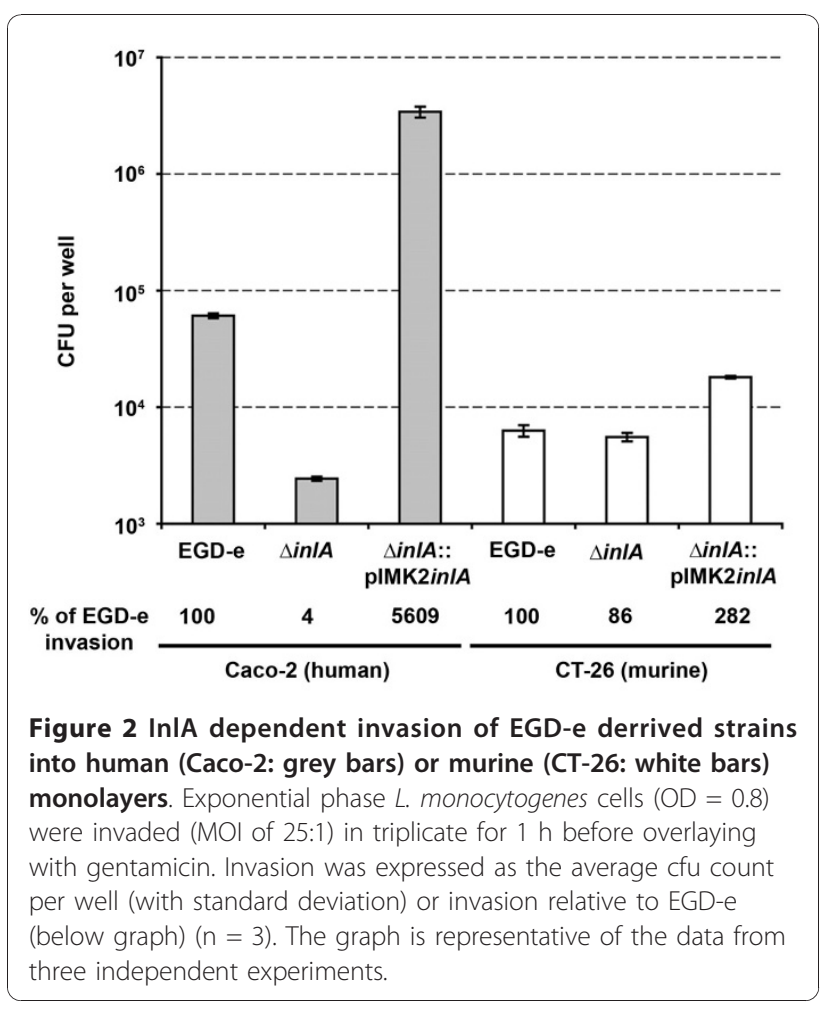

(Figure 1) to produce full length InlA on the surface of $L$. lactis. The system was chosen because production of functional InlA on the cell surface of $L$. lactis had previously been documented [27]. We compared the entry of L. lactis containing vector only (L. lactis-pNZB), producing wild type InlA (L. lactis InlA ${ }^{\mathrm{WT}}$ ) or producing InlA containing the Ser192Asn and Tyr369Ser, but with different codon usage to the previously described murinized InlA ${ }^{\mathrm{m}}$ [17] ( $L$. lactis InlA ${ }^{\left.m_{* *}^{*}\right)}$ into Caco-2 and CT-26 cells. The presence of InlA on the cell surface was confirmed by Western blot analysis (Figure 1b). The level of invasion for L. lactispNZB into Caco-2 cells is similar to that observed for EGD-e $\Delta i n l A$ (Figure 2 and 3). As L. lactis is non invasive, the surviving bacterial cells probably represent bacteria not killed by the gentamicin treatment rather than internalized cells, as documented previously [1]. A similar level of entry into Caco-2 cells was observed for L. lactis InlA ${ }^{\mathrm{WT}}$ and $L$. lactis InlA ${ }^{m_{*}}$, while entry into CT-26 cells was 27-30 fold greater for $L$. lactis InlA ${ }^{m *}$ compared to $L$. lactis InlA ${ }^{\mathrm{WT}}$ (Figure 2).

In contrast to a previous report [11], we observed an increased invasion into a murine cell line by the L. monocytogenes strain over-expressing InlA ${ }^{\mathrm{WT}}$ in contrast to the plasmid only control (Figure 2). A similar trend was observed when the L. monocytogenes InlA over-expressing strain and $\triangle i n l A$ strain were compared (Figure 2) and was also seen in experiments in the L. lactis background (Figure 3 ). These results could be 


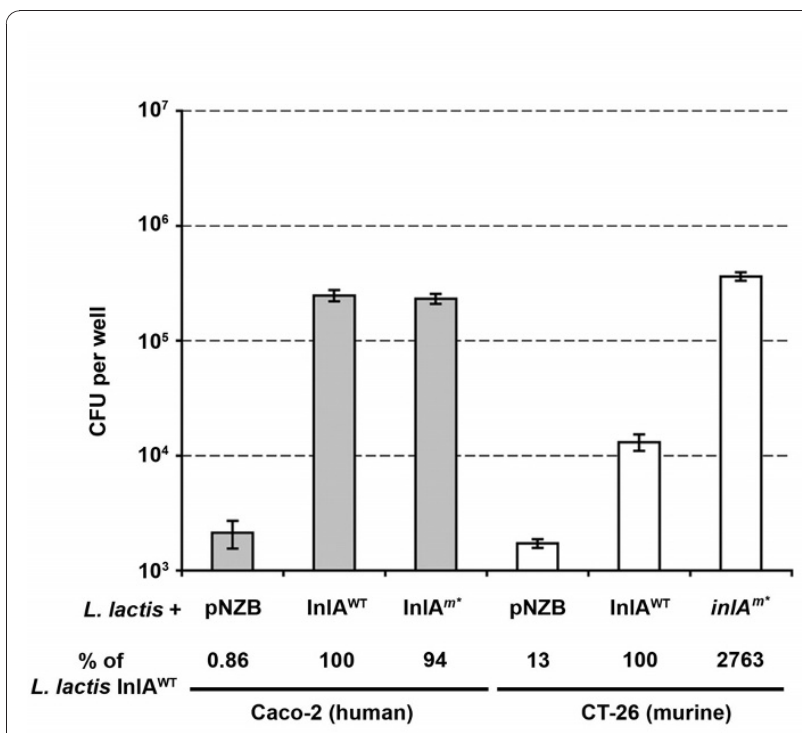

Figure 3 Invasion of $L$. lactis expressing wild type or murinized InIA into Caco-2 (grey bars) or CT-26 (white bars) monolayers. Nisin induced L. lactis cells were invaded (MOI of 25:1) for $1 \mathrm{~h}$ before overlaying with gentamicin. Invasion was expressed as average cfu count (with standard deviation) or invasion relative to $L$. lactis plasmid only (below graph) $(n=3)$. The graph is

representative of the data from three independent experiments.

due to the high level of inlA expression from the Pnis and Phelp promoters, amplifying the differences in InlA on the surface of L. lactis and L. monocytogenes cells (Figure 2 and 3). We interpret these results as evidence of a specific interaction between InlA and a cell surface receptor on CT-26 cells which stimulates bacterial cell entry. To summarise, we have established a gentamicin protection assay, capable of discriminating InlA mediated invasion into a murine cell line.

\section{Generation and screening of a random bank of InIA LRR mutants}

To generate diversity within the inlA gene we applied error prone PCR to the LRR region (between naturally occurring BglII/BstXI sites - Figure 1a). Four separate banks were created containing different levels of mutation frequency, each containing about 40,000 L. lactis clones. Initial assessment by DNA sequencing of ten clones from each bank identified mutations throughout the LRR region with the level of mutation correlating with the concentration of input template DNA for the error prone PCR (data not shown). To identify positive mutations, pools were invaded through CT-26 cells en masse as detailed in Figure 4. Sequential passages through CT-26 cells were required to remove the background functional InlA from the pools (Figure 5). Of the four banks only the highest mutation frequency resulted in an initial recovery below that of wild type InlA, which suggested that a significant number of

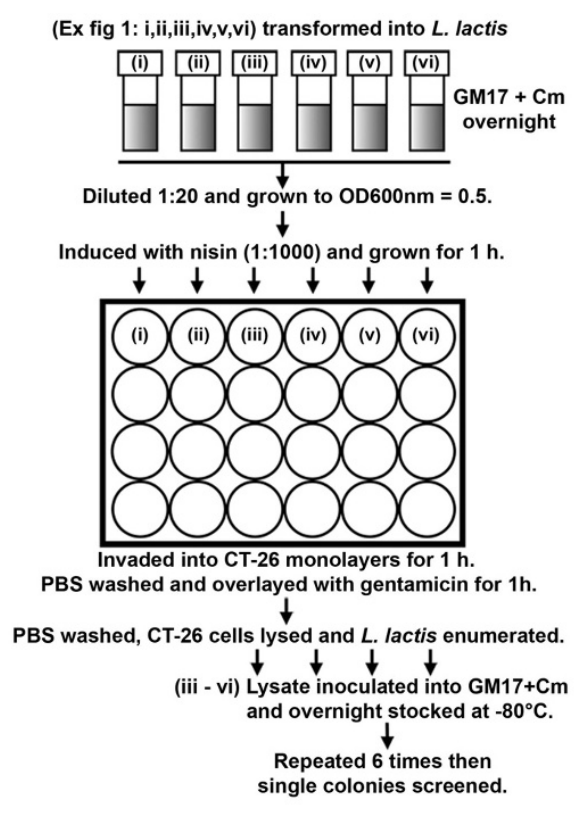

Figure 4 Enrichment protocol for the selection of mutations in InlA conferring enhanced invasion of $L$. lactis into CT-26 cells. Cultures of L. lactis + pNZB containing (i) $i n / A^{W T}$ (ii) $i n / A^{m *}$ or (iii-vi) 4 banks of clones with different levels of mutation in the LRR of in $/ A^{\mathrm{WT}}$ were induced with nisin and assayed for invasion into CT-26 cells by gentamicin protection assay. The residual lysate from invaded CT-26 cells inoculated into GM17 containing $\mathrm{Cm} 5 \mu \mathrm{g} / \mathrm{ml}$ and grown overnight, the culture was then frozen at $-80^{\circ} \mathrm{C}$. The entire process was repeated with the frozen stock serving as the seed for the inoculum.

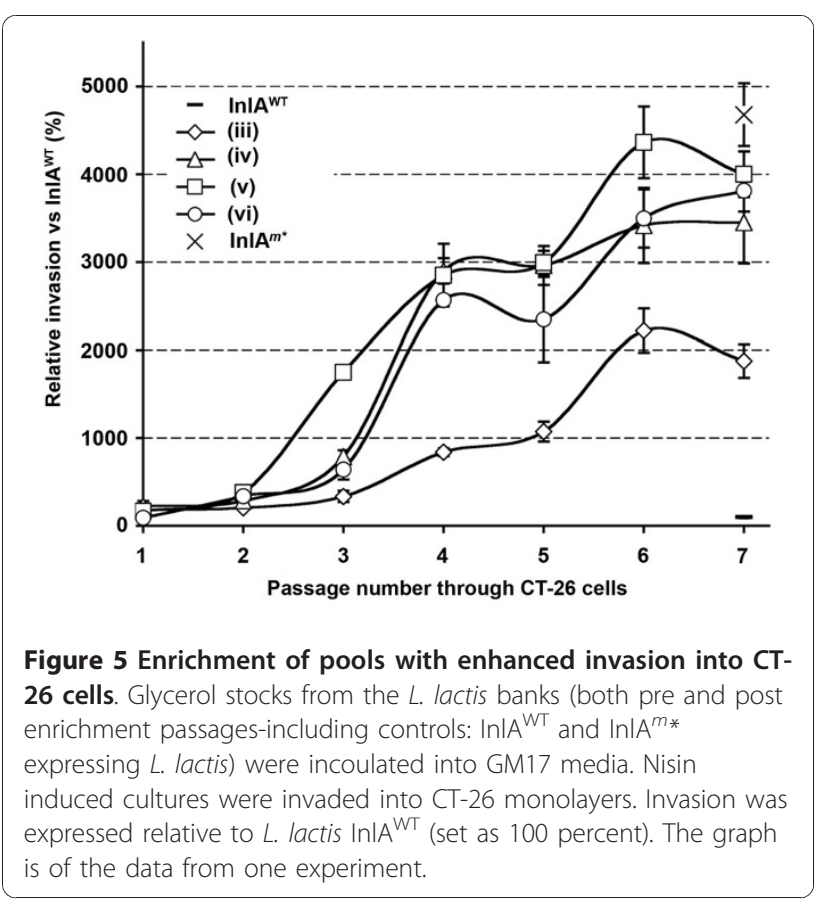


clones contained inactivating mutations. From passage two through six a significant enrichment in positive mutations was observed, with a leveling off at passage seven (Figure 5). From passage six, eight clones from each bank were sequenced (Table 2) and assayed individually using both CT-26 and Caco-2 cells (Figure 6). All clones exhibited enhanced entry into CT-26 cells while no apparent differences for cell entry into Caco-2 cells were observed (compared to L. lactis InlA ${ }^{\mathrm{WT}}$ ). However, no clones were identified which were capable of matching the level of $L$. lactis InlA ${ }^{m *}$ mediated entry into the murine cells. Sequence analysis revealed that 23 of the 32 clones contained amino acid changes in residues involved in direct interaction with CDH1. Of the four banks, only the lowest mutation frequency contained multiple clones with the same mutation (Gln190Leu), with this single amino acid change also found in one clone from an additional bank (Table 2).

\section{Characterization of murinized $L$. monocytogenes: competitive index assays}

Four inlA sequences conferring enhanced invasion into CT-26 cells were selected to be re-created in the chromosome of L. monocytogenes EGD-e. The mutations constituted two single aa changes for EGD-e A (Asn259Tyr) and EGD-e B (Gln190Leu). While three aa changes were introduced into EGD-e C (T164A, K301I, Q303E) and EGD-e D (S173I, L185F, L188I). These mutations were chosen based on the frequency of isolation in L. lactis (EGD-e B and C), the ability to attribute the phenotype to an aa change (EGD-e A) and the isolation of mutations all confined within one LRR (EGD-e D). A fifth strain was also created based on the Lmo-InlA ${ }^{\mathrm{m}}$ mutation [18], except with Listeria optimized codons for 192Asn and 369Ser, and was used as a positive control (EGD-e InlA $\left.{ }^{m *}\right)$. Sequencing confirmed the integrity of the newly introduced mutations, with equivalent levels of InlA expressed on the surface of the strains as compared to EGD-e (assessed by western blot - data not shown). InlA ${ }^{\mathrm{m}}$ strain (termed EGD-e InlA ${ }^{m *}$ ) was compared to the parental EGD-e strain for invasion into Caco-2 and CT-26 monolayers. No differences in invasion (Figure 7a) or adherence (data not shown) were observed to Caco-2 cells, while the invasion of EGD-e InlA $^{m *}$ was significantly higher than EGD-e into CT-26 cells. We then compared the virulence of EGD-e and

Table 2 Supplementary information for Figure 6

\begin{tabular}{|c|c|c|c|c|c|c|c|c|}
\hline Clone & 1 & 2 & 3 & 4 & 5 & 6 & 7 & 8 \\
\hline (iii) Low & T2731 & Q190L & Q190L & Q190L & $\begin{array}{l}\text { Q190L } \\
\text { T229P }\end{array}$ & G303E & Q190L & $\begin{array}{l}\text { Q190L } \\
\text { N386I }\end{array}$ \\
\hline Fold increase vs Wt & 9.44 & 5.82 & 6.98 & 4.15 & 13.23 & 12.12 & 6.10 & 7.94 \\
\hline (iv) Medium & $\begin{array}{l}\text { T164A } \\
\text { K301I } \\
\text { G303E } \\
\text { T399I }\end{array}$ & $\begin{array}{l}\text { L86F } \\
\text { N143K } \\
\text { P159A } \\
\text { Q196L } \\
\text { K218M } \\
\text { V224A } \\
\text { G303E } \\
\text { Q306H }\end{array}$ & $\begin{array}{l}\text { Q190L } \\
\text { L329Q } \\
\text { S470C }\end{array}$ & $\begin{array}{l}\text { T164A } \\
\text { K301I } \\
\text { G303E }\end{array}$ & $\begin{array}{l}\text { N259Y } \\
\text { T399| }\end{array}$ & $\begin{array}{l}\text { Q190L } \\
\text { G248R }\end{array}$ & $\begin{array}{l}\text { F193Y } \\
\text { K301E } \\
\text { N413Y } \\
\text { K507I }\end{array}$ & $\begin{array}{l}\text { T164A } \\
\text { K301I } \\
\text { G303E }\end{array}$ \\
\hline Fold increase vs Wt & 3.25 & 9.31 & 7.79 & 6.85 & 8.14 & 6.57 & 4.05 & 10.08 \\
\hline (v) High & $\begin{array}{l}\text { L149M } \\
\text { N259Y }\end{array}$ & $\begin{array}{l}\text { Q190L } \\
\text { S223C } \\
\text { N252Y } \\
\text { I351T }\end{array}$ & $\begin{array}{l}\text { S173I } \\
\text { G303E } \\
\text { T446A } \\
\text { D449H }\end{array}$ & $\begin{array}{l}\text { S173I } \\
\text { T268I } \\
\text { G303E } \\
\text { T446A } \\
\text { D449H }\end{array}$ & $\begin{array}{l}\text { Q190L } \\
\text { S223C } \\
\text { N252Y } \\
\text { I351T }\end{array}$ & N259Y & $\begin{array}{l}\text { N239D } \\
\text { S311C } \\
\text { N325D }\end{array}$ & $\begin{array}{l}S 173 I \\
L 185 F \\
L 188 I\end{array}$ \\
\hline Fold increase vs Wt & 23.21 & 15.89 & 8.64 & 19.31 & 9.08 & 16.36 & 8.24 & 15.42 \\
\hline (vi) Very High & $\begin{array}{l}\text { Q190L } \\
\text { A270G } \\
\text { K301G }\end{array}$ & $\begin{array}{l}\text { V123A } \\
\text { Q190L } \\
\text { P290Q } \\
\text { N349D }\end{array}$ & Q190L & $\begin{array}{l}\text { Q196K } \\
\text { P290S } \\
\text { L404S } \\
\text { N413Y } \\
\text { D457V }\end{array}$ & $\begin{array}{l}\text { N130I } \\
\text { F150V } \\
\text { L203F } \\
\text { Y369F } \\
\text { N381I } \\
\text { S487N }\end{array}$ & $\begin{array}{l}\text { L294V } \\
\text { S308R } \\
\text { Y369S } \\
\text { N381I } \\
\text { S487N }\end{array}$ & $\begin{array}{l}\text { L122I } \\
\text { S292T } \\
\text { E330V } \\
\text { I458V }\end{array}$ & $\begin{array}{l}\text { Q190L } \\
\text { D199V } \\
\text { S377N } \\
\text { P444S } \\
\text { K495N }\end{array}$ \\
\hline Fold increase vs Wt & 4.14 & 9.33 & 6.96 & 8.71 & 9.56 & 7.12 & 7.51 & 9.33 \\
\hline
\end{tabular}

Mutations identified in the Bglll/BstXI fragment of pNZBinlA (iii-vi) and the invasion increase into CT-26 cells versus $L$. lactis InIA ${ }^{\text {WT }}$. The amino acid mutations identified which involved in the interaction between InIA ${ }^{\mathrm{WT}}$ and $\mathrm{hCDH} 1$ are highlighted in bold.

Details highlighted in bold and italics are mutations recombined in the chromosome of EGD-e.

L. lactis InIA site directed mutants with fold invasion increase into CT-26 cells vs L. lactis InIA ${ }^{\text {WT }}$ in brackets: S192N (21), Y369 S (20), S192N+Y369 S (30).

Below: Amino acids in InlA ${ }^{\mathrm{WT}}$ which interact with $\mathrm{hCDH} 1$ and amino acid changes identified from error prone PCR screen. R85, N104: D Q*, N107, F150: V, E170, E172: T*, Q190: L, S192, R211, D213, I235, T237, E255, N259: Y, K301: I E G, N321: Y, E323, N325: D, E326, Y343, T345, Y347, F348, R365, F367, Y369: F S, W387, S389. * N104 and E172 mutations were found from additional screens and sequencing. 


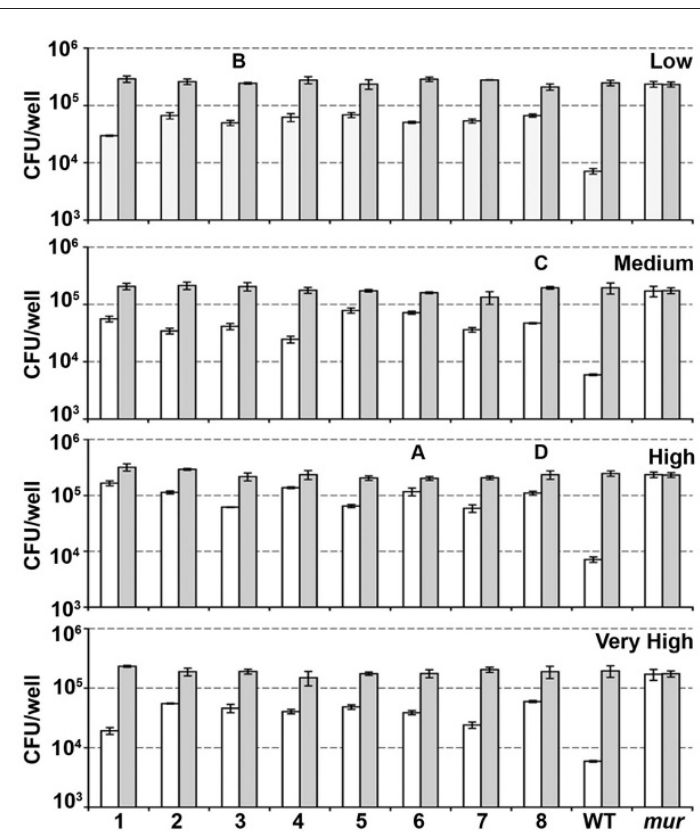

Figure 6 Invasion attributes of individual L. lactis clones post CT-26 enrichment (passage 6) into Caco-2 (grey bars) or CT-26 (white bars) cells. From each of the four banks, eight clones were picked and invaded with invasion expressed as the average (with standard deviation) from triplicate wells. Sequnce data of the clones is presented in Table 2. Letters above bars indicate sequences that were subsequently used to recreate into the $L$. monocytogenes chromosome. The controls $\operatorname{InI} \mathrm{A}^{\mathrm{WT}}(\mathrm{WT})$ and $\operatorname{In|} \mathrm{A}^{m *}$ (mur) expressing $L$. lactis were included for comparison. The graph is of the data from one experiment.

EGD-e InlA ${ }^{m *}$ by competitive index (CI) assays via the intravenous (i.v.) (Figure 7b) or intragastric (i.g.) (Figure $7 \mathrm{c})$ route in Balb/c mice. For i.v. inoculated mice, no differences in the kinetics of infection were observed for either strain (Figure 7b). This confirms that the two amino acid changes in InlA ${ }^{\mathrm{m}}$ do not impact on the virulence of EGD-e InlA ${ }^{m *}$ once the gastrointestinal tract is bypassed. However, EGD-e InlA ${ }^{m_{*}}$ was significantly more virulent when infected by the i.g. route, with higher counts obtained from livers and spleens and a significantly higher CI value $(\mathrm{p}<0.001)$ for both day two (Liver 28.9, Spleen 10.6) and day three (Liver 24.9, Spleen 7.7 - Figure 7c). Neither strain was recovered form the liver nor spleen at day one post infection. Subsequent competitive index experiments were conducted by the i.g. route comparing EGD-e InlA ${ }^{m *}$ against the strains expressing the InlA mutations identified by the CT-26 cell screen (Figure 7d). Of the four recreated strains, only EGD-e A (N259Y) gave a higher CI than EGD-e in the liver (0.19 vs 0.05$)$ whereas identical values $(0.12)$ were obtained for the spleens. Further experimentation will be required to access the contribution of the N259Y mutation, and it would be intriguing to see if the recombination of this mutation into EGD-e InlA ${ }^{m *}$ would further enhance murine pathogenicity. It is interesting to note that the strain in which InlA $^{\mathrm{m}}$ (with Listeria optimized codons for 192Asn and 369Ser) was recreated (EGD-e InlA ${ }^{m *}$ ) did not exhibit enhanced invasion or adhesion to Caco-2 cells, which is a marker for human virulence, in contrast to the previously published results [17]. To further explore the progression of i.g. infection, we repeated the Balb/c inoculations with either EGD-e or EGD-e InlA ${ }^{m *}$ tagged with a constitutive bioluminescent lux marker and mice were imaged for bioluminescence on each subsequent day [18]. The EGD-e InlA ${ }^{m *}$ strain exhibited uniform clinical signs of $L$. monocytogenes infection by day 2 [28], while these characteristics were absent from the EGD-e group even prior to sacrifice at day 3. Consistent with the clinical scores very little light was observed from the EGD-e group, while increasing light levels were obtained from the EGD-e InlA ${ }^{m *}$ group on days 1 and 2, with a distinct foci evident in the abdomen in all 5 mice by day 3 (Figure 8a). Upon ex vivo imaging of the livers, a low signal was present in the gall bladder in 3 of the 5 EGD-e infected mice, whereas a much stronger signal was found from the gall bladders of all EGD-e InlA ${ }^{m *}$ (5 out of 5 ) infected mice, with infection across the liver also observed (Figure 8a). The EGD-e InlA ${ }^{m *}$ infected gall bladders were also found to be to twice the size of the EGD-e group. Further work is necessary to determine the exact extent of gall bladder colonization in these animals relative to hepatocyte infection. Enumeration of the livers and spleens confirmed that the EGD-e InlA ${ }^{m *}$ strain produced highly reproducible i.g. infections, with the levels recovered comparable to day three i.v. infections in the liver (Figure $8 \mathrm{~b}$ ). A much larger degree of variation was observed in the EGD-e group, with statistically significant differences in bacterial counts observed between the two strains (Figure 8b). The mechanism of gall bladder colonization is currently unknown $[29,30]$ and warrants further investigation. The EGD-e InlA ${ }^{m_{*}}$ strain is capable of establishing highly reproducible colonization of the gall bladder upon i.g. inoculation. This strain will be extremely useful in examining factors required for gastrointestinal transit and gall bladder colonization.

\section{Discussion}

It is now well established that the murine model of listeriosis is limited by a poor interaction between the bacterial invasion protein InlA and its host ligand mCDH1. This is in direct contrast, to the efficient interaction between InlA and hCDH1. The discrepancy is due to a glutamate at residue 16 in mouse (and rat) E-cadherin rendering these host species relatively resistant to 


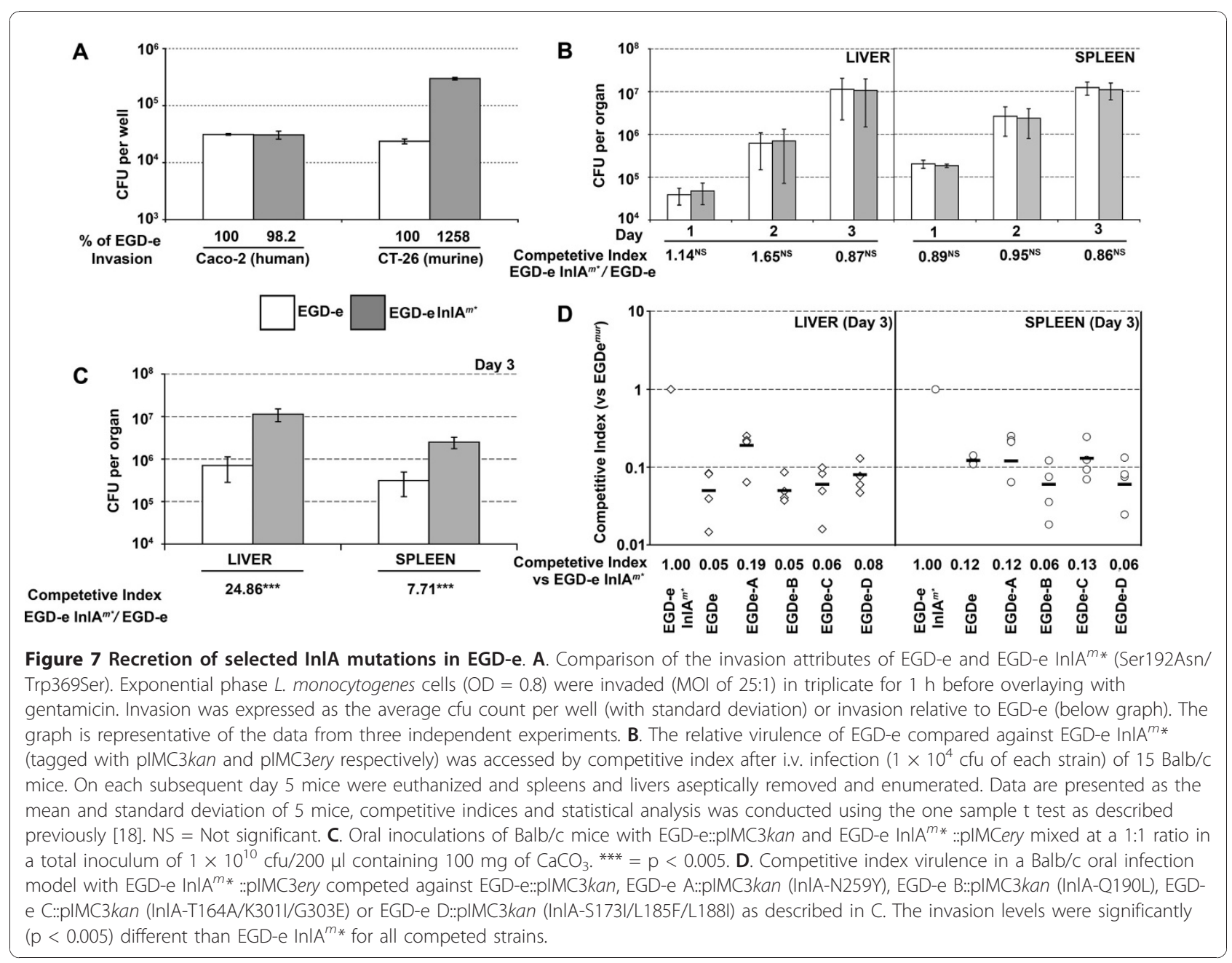

infection by the oral route and limiting their use as laboratory models for certain $L$. monocytogenes-mediated disease processes [11]. Recent studies have developed an engineered mouse strain expressing 'humanized' E-cadherin for studies of oral and fetoplacental listeriosis [14]. An alternative approach has utilized structure-based engineering to 'murinize' the bacterial InlA protein in order to increase affinity for murine E-cadherin [17]. This approach has provided key insights into the interaction between InlA and CDH1. While murinization was highly successful, we reasoned that additional points of contact may also improve the interaction with $\mathrm{mCDH} 1$. We therefore developed a system to select random mutations in InlA that enhance invasion of murine cells in order to identify novel amino acid interactions and to determine if 'murinization' of the strain can be improved.

L. lactis was used as a surrogate host for this process in order to prevent generation of Listeria mutants with increased affinity for human cells. Previous research had shown that heterologous expression of InlA from the native PinlA promoter in L. lactis could stimulate invasion into cultured human colonic enterocytes and guinea pig enterocytes in an oral infection model [27]. Additional properties of $L$. lactis such as high transformation efficiency $\left(4 \times 10^{4} \mathrm{cfu}\right.$ for ligations) allowed us to generate multiple random libraries of substantial size and enabled the direct transformation of SDM constructs. Also the nisin inducible system enabled a high level of InlA expression on the surface of L. lactis in a background with relatively few sortase A anchored proteins.

The ability of L. lactis InlA $^{m *}$ to facilitate uptake into murine cells encouraged us to use multiple rounds of en masse enrichment of InlA mutant libraries through CT26 cells. The cumulative results from each passage showed a continued improvement in the invasion efficiency, suggestive of an enrichment of positive clones. A surprising level of diversity in InlA clones was apparent (across the 4 banks) with 25 of the 32 clones analyzed exhibiting unique sequences. Only bank iii with the lowest frequency of mutations exhibited a degree of clonality (4/8 were Q190L). This suggests that we have not 


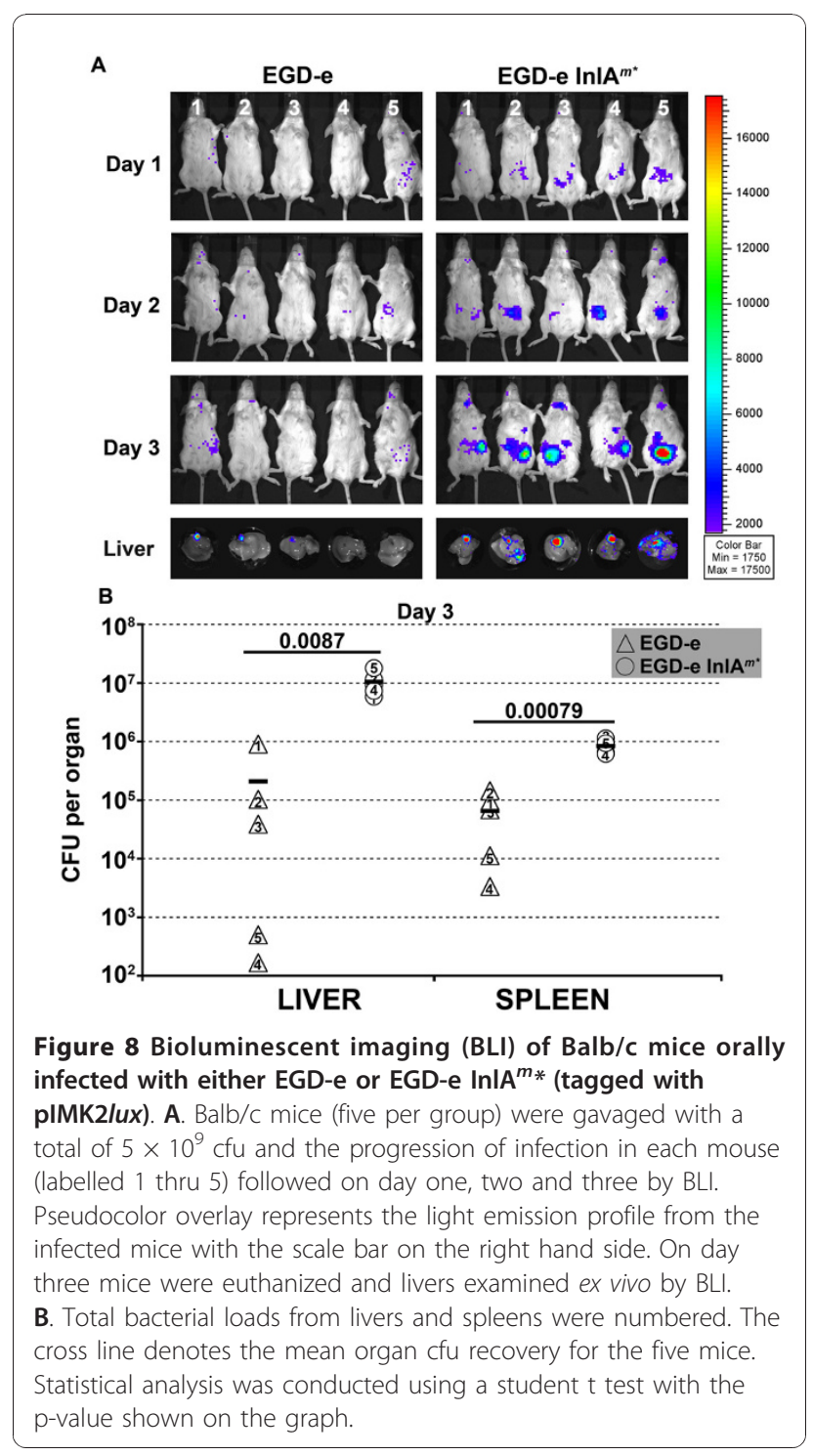

yet uncovered the full complement of mutations within the banks which confer enhanced invasion capabilities.

Directed evolution of the inlA gene has the potential to uncover mutations not predicted by a structure-based approach (Table 2). With respect to the Q190L mutation the glutamine at residue 190 found on LRR 6 within the hydrophobic pocket, and forms a hydrogen bond to proline 16 in hCDH1. The change to leucine may affect the pocket and improve access of glutamic acid 16 in $\mathrm{mCDH} 1$. Of all the single amino acid changes, the N259Y mutation exhibited the single greatest invasion increase into CT-26 cells. Combining this mutation with either T399I or L149 M was shown to reduce or enhance invasion, respectively, with the negative effect of the T399I confirmed by the reduction in invasion efficiency observed when combined with additional positive mutations (bank IV, clone 8 versus bank
IV, clone 1-Table 2). Further biochemical studies will be required to identify the role these mutations play to enhance the interaction with $\mathrm{mCDH} 1$. The previously identified single aa changes at residues 192 and 369 [17] each increased invasion $\sim 20$ fold, whereas the combined $192+369$ mutations increased invasion $\sim 30$ fold. The identical aa change at residue 369 was also isolated from our error prone PCR bank. However, this clone contained additional mutations that resulted in a reduced level of invasion compared to the 369 single mutant.

The CDH1 interacting amino acids appear to be highly conserved and recalcitrant to change [31]. From a collection of $101 \mathrm{inlA}$ gene sequences mapped onto the InlA crystal structure [32], three naturally occurring InlA variants were identified which could potentially mediate an interaction with hCDH1, with one (Lys301Glu) also identified through the random mutagenesis approach in our study. However, while all mutants containing this residue had a positive effect on invasion into CT-26 cells, the exact contribution of this residue could not be assessed as additional mutations were present in all clones. Further analysis of individual clones from each bank or the application of additional selection is required due to the diversity uncovered ( 25 of the 32 clones analyzed were different). This diversity and the enhanced invasion of all the clones examined confirms that amino acids additional to the ones previously examined [17] can modulate the affinity for CDH1.

Despite the analysis of 32 clones from our enriched bank of InlA variants, we failed to detect mutations that yielded invasion rates comparable to the murinized InlA described by Wollert and coworkers [17]. In terms of developing usable models of murine listeriosis the approach of 'murinizing' the bacterial strain arguably has a number of benefits over the development of humanized mouse lines. Development of the modified bacterium will permit utilization of this strain in existing mouse lines (including existing knock-out murine models) and distribution of the murinized strain is relatively straightforward, as is the creation of new mutations in the EGD-e InlA ${ }^{m *}$ background. However, the 2-fold enhanced adherence and invasion to human (Caco-2) cells of the L. monocytogenes Lmo-InlA ${ }^{\mathrm{m}}$ [17] could be a potential cause for concern as it is suggestive of a slight enhancement of virulence towards humans. The procedure used to create that strain required multiple prolonged incubations at $42^{\circ} \mathrm{C}[17,33]$. It has been recently shown that high temperature growth of L. monocytogenes can induce spontaneous mutation, suggesting that high temperature growth should be minimized to avoid the acquisition of secondary mutations [34]. We re-created the InlA mutations described by Wollert et al., [17] to create EGD-e InlA ${ }^{m *}$ using only two temperature shifts to $37^{\circ} \mathrm{C}$ and six passages under non-selective 
conditions [20]. Another difference between the LmoInlA $^{\mathrm{m}}$ and EGD-e InlA ${ }^{m *}$ strain were the nucleotide changes made to create the mutated amino acids. In the EGD-e InlA ${ }^{m *}$ strain the two codons were chosen based on the codon usage from genome analysis, with the most commonly used triplets applied. In each case usage was $50 \%$ higher than the one used in Lmo-InlA ${ }^{\mathrm{m}}$. For the asparagine 192, AAT compared to the AAC codon was chosen (31.8 vs 14.4 per 1000 codons). While for serine 369 TCT compared to TCG codon was chosen (12.8 vs 6.2 per 1000 codons). The invasion data for Lmo-InlA ${ }^{\mathrm{m}}$ agreed with the biophysical characterization which showed an enhanced interaction for InlA with CDH1 [35] however as recently shown, synonymous mutations leading to mRNA sequence changes can also affect substrate specificity or protein activity [36]. To access the role of codon usage or strain background, competitive index experiments will need to be conducted to directly compare Lmo-InlA ${ }^{\mathrm{m}}$ with EGD-e $\operatorname{InlA}^{m *}$.

\section{Conclusions}

The research presented here generated random InlA variants with enhanced invasion into the CT-26 cell line most likely through an increased affinity for mCDH1. Novel mutations in InlA were readily identified from the random mutagenesis approach and a number (including the N259Y mutation) are worthy of further study. The approach used here indicates that other random or targeted mutagenesis strategies may uncover mutations that further enhance protein-ligand binding. In particular we suggest that screening approaches such as biopanning [37] using the first extra cellular domain of mCDH1 as bait or a site-saturation mutagenesis approach (the analysis of all amino acid combinations at a single residue) [38] may uncover further potential interactions. We have demonstrated that the newly created strain, EGD-e InlA ${ }^{m *}$ does not have an enhanced affinity for human cells (unlike the predecessor EGD$\mathrm{InlA}^{\mathrm{m}}$ ) while displaying highly reproducible oral infections in the mouse model. The use of this murinized $L$. monocytogenes strain will prove a useful tool in analysing the gastrointestinal phase of listeriosis. The additional residues identified here as playing a role in InlA:: $\mathrm{CDH} 1$ interactions will inform our ongoing efforts to create safer 'murinised' versions of L. monocytogenes which will help us to combat this often fatal pathogen.

\section{Acknowledgements}

The authors would like to thank Richard O'Kennedy and Stephen Harty for generously supplying the InIA monoclonal antibody. We would like to acknowledge the funding received from the Irish Government under the National Development Plan 2000-2006 and the funding of the Alimentary Pharmabiotic Centre by the Science Foundation of Ireland Centres for Science Engineering and Technology (CSET) programme.

\section{Author details}

${ }^{1}$ Alimentary Pharmabiotic Centre \& Department of Microbiology, University College Cork, Western Road, Cork, Ireland. ${ }^{2}$ Alimentary Pharmabiotic Centre, Department of Microbiology \& School of Pharmacy, University College Cork Western Road, Cork, Ireland. '3School of Genetics and Microbiology,

Department of Microbiology, Trinity College Dublin, Ireland.

\section{Authors' contributions}

All authors read and approved the final manuscript. IRM devised the study, carried out the experimental work and wrote the manuscript; PGC carried out murine infection work; $\mathrm{CH}$ and CGMG devised and guided the study and helped to draft the manuscript.

Received: 14 May 2010 Accepted: 13 December 2010

Published: 13 December 2010

\section{References}

1. Gaillard $J L$, Berche P, Frehel $C$, Gouin E, Cossart P: Entry of $L$. monocytogenes into cells is mediated by internalin, a repeat protein reminiscent of surface antigens from gram-positive cocci. Cell 1991, 65:1127-1141.

2. Bierne $H$, Sabet $C$, Personnic N, Cossart P: Internalins: a complex family of leucine-rich repeat-containing proteins in Listeria monocytogenes. Microbes Infect 2007, 9:1156-1166.

3. Mengaud J, Lecuit M, Lebrun M, Nato F, Mazie JC, Cossart P: Antibodies to the leucine-rich repeat region of internalin block entry of Listeria monocytogenes into cells expressing E-cadherin. Infect Immun 1996, 64:5430-5433.

4. Lecuit M, Ohayon $H$, Braun L, Mengaud J, Cossart P: Internalin of Listeria monocytogenes with an intact leucine-rich repeat region is sufficient to promote internalization. Infect Immun 1997, 65:5309-5319.

5. Mengaud J, Ohayon H, Gounon P, Mege R-M, Cossart P: E-cadherin is the receptor for internalin, a surface protein required for entry of $L$. monocytogenes into epithelial cells. Cell 1996, 84:923-932.

6. Guilford P, Hopkins J, Harraway J, McLeod M, McLeod N, Harawira P, Taite H, Scoular R, Miller A, Reeve AE: E-cadherin germline mutations in familial gastric cancer. Nature 1998, 392:402-405.

7. Pron B, Boumaila C, Jaubert F, Sarnacki S, Monnet JP, Berche P, Gaillard JL: Comprehensive study of the intestinal stage of listeriosis in a rat ligated ileal loop system. Infect Immun 1998, 66:747-755.

8. Marco AJ, Altimira J, Prats N, Lopez S, Dominguez L, Domingo M, Briones V: Penetration of Listeria monocytogenes in mice infected by the oral route. Microb Pathog 1997, 23:255-263.

9. Racz P, Tenner K, Mero E: Experimental Listeria enteritis. I. An electron microscopic study of the epithelial phase in experimental listeria infection. Lab Invest 1972, 26:694-700.

10. Gaillard JL, Finlay BB: Effect of cell polarization and differentiation on entry of Listeria monocytogenes into the enterocyte-like Caco-2 cell line. Infect Immun 1996, 64:1299-1308.

11. Lecuit M, Dramsi S, Gottardi C, Fedor-Chaiken M, Gumbiner B, Cossart P: A single amino acid in E-cadherin responsible for host specificity towards the human pathogen Listeria monocytogenes. EMBO J 1999, 18:3956-3963.

12. Lecuit M, Vandormael-Pournin S, Lefort J, Huerre M, Gounon P, Dupuy C, Babinet C, Cossart P: A transgenic model for listeriosis: role of internalin in crossing the intestinal barrier. Science 2001, 292:1722-1725.

13. Pentecost M, Otto G, Theriot JA, Amieva MR: Listeria monocytogenes invades the epithelial junctions at sites of cell extrusion. PLOS Pathog 2006, 2:e3.

14. Disson O, Grayo S, Huillet E, Nikitas G, Langa-Vives F, Dussurget $O$, Ragon M, Le Monnier A, Babinet C, Cossart P, Lecuit M: Conjugated action of two species-specific invasion proteins for fetoplacental listeriosis. Nature 2008, 455:1114-1118.

15. Schubert WD, Urbanke C, Ziehm T, Beier V, Machner MP, Domann E, Wehland J, Chakraborty T, Heinz DW: Structure of internalin, a major invasion protein of Listeria monocytogenes, in complex with its human receptor E-cadherin. Cell 2002, 111:825-836.

16. Khelef N, Lecuit M, Bierne $H$, Cossart P: Species specificity of the Listeria monocytogenes InIB protein. Cell Microbiol 2006, 8:457-470.

17. Wollert T, Pasche B, Rochon M, Deppenmeier S, van den Heuvel J, Gruber AD, Heinz DW, Lengeling A, Schubert WD: Extending the host 
range of Listeria monocytogenes by rational protein design. Cell 2007, 129:891-902.

18. Monk IR, Casey PG, Cronin M, Gahan CG, Hill C: Development of multiple strain competitive index assays for Listeria monocytogenes using PIMC; a new site-specific integrative vector. BMC Microbiol 2008, 8:96.

19. Holo H, Nes IF: High-Frequency Transformation, by Electroporation, of Lactococcus lactis subsp. cremoris Grown with Glycine in Osmotically Stabilized Media. Appl Environ Microbiol 1989, 55:3119-3123.

20. Monk IR, Gahan CG, Hill C: Tools for functional postgenomic analysis of Listeria monocytogenes. Appl Environ Microbiol 2008, 74:3921-3934.

21. Wang W, Malcolm BA: Two-stage PCR protocol allowing introduction of multiple mutations, deletions and insertions using QuikChange SiteDirected Mutagenesis. Biotechniques 1999, 26:680-682.

22. Monk IR, Cook GM, Monk BC, Bremer PJ: Morphotypic conversion in Listeria monocytogenes biofilm formation: biological significance of rough colony isolates. Appl Environ Microbiol 2004, 70:6686-6694.

23. Hearty S, Leonard P, Quinn J, O'Kennedy R: Production, characterisation and potential application of a novel monoclonal antibody for rapid identification of virulent Listeria monocytogenes. J Microbiol Methods 2006, 66:294-312.

24. Corbett TH, Griswold DPJ, Roberts BJ, Peckham JC, Schabel FMJ: Tumor induction relationships in development of transplantable cancers of the colon in mice for chemotherapy assays, with a note on carcinogen structure. Cancer Res 1975, 35:2434-2439.

25. Brattain MG, Strobel-Stevens J, Fine D, Webb M, Sarrif AM: Establishment of mouse colonic carcinoma cell lines with different metastatic properties. Cancer Res 1980, 40:2142-2146.

26. Mierau I, Kleerebezem M: 10 years of the nisin-controlled gene expression system (NICE) in Lactococcus lactis. Appl Microbiol Biotechnol 2005, 68:705-717.

27. Guimaraes VD, Gabriel JE, Lefevre F, Cabanes D, Gruss A, Cossart P, Azevedo V, Langella P: Internalin-expressing Lactococcus lactis is able to invade small intestine of guinea pigs and deliver DNA into mammalian epithelial cells. Microbes Infect 2005, 7:836-844.

28. Bron PA, Monk IR, Corr SC, Hill C, Gahan CG: Novel luciferase reporter system for in vitro and organ-specific monitoring of differential gene expression in Listeria monocytogenes. Appl Environ Microbiol 2006, 72:2876-2884

29. Hardy J, Francis KP, DeBoer M, Chu P, Gibbs K, Contag CH: Extracellular replication of Listeria monocytogenes in the murine gall bladder. Science 2004, 303:851-853

30. Hardy J, Margolis JJ, Contag CH: Induced biliary excretion of Listeria monocytogenes. Infect Immun 2006, 74:1819-1827.

31. Orsi RH, Ripoll DR, Yeung M, Nightingale KK, Wiedmann M: Recombination and positive selection contribute to evolution of Listeria monocytogenes inlA. Microbiology 2007, 153:2666-2678.

32. Wollert T: Rational Pathogen Design: Extending the Host Range of Listeria monocytogenes by Thermodynamically Re-engineering the Internalin/E-Cadherin Interface. PhD thesis, Technical University CaroloWilhelmina, Braunschweig 2007.

33. Lingnau A, Domann E, Hudel M, Bock M, Nichterlein T, Wehland J, Chakraborty T: Expression of the Listeria monocytogenes EGD inlA and inlB genes, whose products mediate bacterial entry into tissue culture cell lines, by PrfA-dependent and -independent mechanisms. Infect Immun 1995, 63:3896-3903.

34. Vega Y, Rauch M, Banfield MJ, Ermolaeva S, Scortti M, Goebel W, VazquezBoland JA: New Listeria monocytogenes prf $A^{*}$ mutants, transcriptional properties of $\operatorname{PrfA}^{*}$ proteins and structure-function of the virulence regulator PrfA. Mol Microbiol 2004, 52:1553-1565.

35. Wollert T, Heinz DW, Schubert WD: Thermodynamically reengineering the listerial invasion complex InIA/E-cadherin. Proc Natl Acad Sci USA 2007, 104:13960-13965.

36. Angov E, Hillier CJ, Kincaid RL, Lyon JA: Heterologous protein expression is enhanced by harmonizing the codon usage frequencies of the target gene with those of the expression host. PLOS ONE 2008, 3:e2189.

37. Hoogenboom HR: Selecting and screening recombinant antibody libraries. Nat Biotechnol 2005, 23:1105-1116.

38. Field D, Connor PM, Cotter PD, Hill C, Ross RP: The generation of nisin variants with enhanced activity against specific Gram-positive pathogens. Mol Microbiol 2008, 69:218-230.
39. Glaser P, Frangeul L, Buchrieser C, Rusniok C, Amend A, Baquero F, Berche P, Bloecker H, Brandt P, Chakraborty T, Charbit A, Chetouani F, Couve E, de Daruvar A, Dehoux P, Domann E, Dominguez-Bernal G, Duchaud E, Durant L, Dussurget O, Entian KD, Fsihi H, Garcia-del Portillo F, Garrido P, Gautier L, Goebel W, Gomez-Lopez N, Hain T, Hauf J, Jackson D, Jones LM, Kaerst U, Kreft J, Kuhn M, Kunst F, Kurapkat G, Madueno E, Maitournam A, Vicente JM, Ng E, Nedjari H, Nordsiek G, Novella S, de Pablos B, Perez-Diaz JC, Purcell R, Remmel B, Rose M, Schlueter T, Simoes N, Tierrez A, Vazquez-Boland JA, Voss H, Wehland J, Cossart P: Comparative genomics of Listeria species. Science 2001, 294:849-852.

40. Leenhouts K, Venema G, Kok J: A lactococcal pWV01-based integration toolbox for bacteria. Methods in Cell Science 1998, 20:35-50.

41. Maguin E, Duwat P, Hege T, Ehrlich D, Gruss A: New thermosensitive plasmid for Gram-positive bacteria. J Bacteriol 1992, 174:5633-5638.

\section{doi:10.1186/1471-2180-10-318}

Cite this article as: Monk et al:: Directed evolution and targeted mutagenesis to murinize listeria monocytogenes internalin A for enhanced infectivity in the murine oral infection model. BMC Microbiology 2010 10:318.

\section{Submit your next manuscript to BioMed Central and take full advantage of:}

- Convenient online submission

- Thorough peer review

- No space constraints or color figure charges

- Immediate publication on acceptance

- Inclusion in PubMed, CAS, Scopus and Google Scholar

- Research which is freely available for redistribution

Submit your manuscript at www.biomedcentral.com/submit
C Biomed Central 